\title{
Interacción medicamentosa en Geriatría
}

\author{
TEODORO OSCANOA \\ Médico del Servicio de Geriatría del Hospital Nacional Guillermo Almenara Irigoyen.. Lima. Perú. \\ Profesor de la Facultad de Medicina de la Universidad Mayor de San Marcos. \\ Miembro del Instituto de Gerontologia de la Universidad Peruana Cayetano Heredia.
}

\begin{abstract}
Resumen
Los pacientes adultos mayores tienen tres características principales que lo diferencian de otros grupos etáreos: polipatología, polifarmacia y cambios fisiológicos relacionados con el envejecimiento que alteran la farmacocinética y farmacodinámica de los medicamentos. Estos tres factores hacen que la interacción medicamentosa (interacción fármaco-fármaco) se manifieste con mayor frecuencia y severidad en este grupo etáreo, actualmente forma parte de los problemas relacionados con medicamentos (PRM) en el adulto mayor, que necesitan ser estudiados en su epidemiología así como en las estrategias adecuadas para combatirla. El uso adecuado de los fármacos y la monitorización estrecha de éstos son esenciales en la prevención de las reacciones adversas medicamentosas producidas por las interacciones farmacológicas.
\end{abstract}

Palabras clave: Interacciones de drogas; anciano; envejecimiento; monitoreo de drogas; sistemas de registro de reacción adversa a medicamentos; geriatría.

\section{Drug interaction in Geriatrics}

Abstract

Elderly patients have three main characteristics that differentiate them from other population groups: polypathology, polypharmacy and physiologic changes related with aging that alter drugs' pharmocokinetic and pharmacodynamic. These three factors increase drug-drug interactions (DDI) in frequency and severity. Drug-drug interactions are part of drug related problems (DRP) in elderly patient that need to be studied in regards to its epidemiology and adequate strategies to prevent it. Prudent use of medications and vigilant drug monitoring are essential to avoid adverse drug reactions caused by drug-drug interactions.

Key words: Drug interactions; aged; aging; drug monitoring; adverse drug reaction reporting system; geriatrics.

\section{INTRODUCCIÓN}

Entre los años 1998 y 2000 se retiró del mercado farmacéutico los siguientes medicamentos: mibefradil, terfenadina, astemizol y cisaprida. ¿Cuál fue la causa en común? Graves reacciones adversas producidas por interacciones con otros medicamentos, que,

Correspondencia:

Dr. Teodoro J. Oscanoa

Servicio de Geriatría Hospital Almenara

Av Grau 800 La Victoria. Lima 13, Perú.

E-mail: toscanoa@sanfer.unmsm.edu.pe en su mayor parte, fueron detectados en el periodo postmarketing, es decir, después de haber sido ampliamente comercializados en el mundo $\left(^{1,2}\right)$. ¿Por qué siempre es necesario asociar carbidopa-levodopa, amoxicilinaclavulanato potásico o imipinem-cilastatina? Porque la interacción farmacológica en estos casos es sinergística y la asociación es benéfica para el paciente. La interacción farmacológica se puede mover entre estos dos extremos, es decir, desde la generación de un riesgo innecesario para el paciente hasta la necesidad de esa interacción para que la terapia sea efectiva. 
Los pacientes adultos mayores tienen tres características principales que lo diferencian de otros grupos etáreos: polipatología, polifarmacia y cambios fisiológicos relacionados con el envejecimiento, que alteran la farmacocinética y farmacodinámica de los medicamentos. Estos tres factores contribuyen a que la interacción medicamentosa que puede pasar desapercibida en un paciente joven, en el adulto mayor se manifieste como una reacción adversa severa, que, en el mejor de los casos, si es detectada como tal podrá corregirse, pero la mayor parte de veces es interpretada erróneamente como empeoramiento de la enfermedad, pobre adherencia al tratamiento $\left({ }^{3}\right)$ o inefectividad de alguno de los fármacos interactuantes.

La interacción farmacológica forma parte de los problemas relacionados con medicamentos (PRM) en el adulto mayor que necesita ser estudiado en su epidemiología así como en las estrategias adecuadas para combatirla. Realmente, los fármacos pueden interaccionar con alimentos, suplementos nutricionales, productos de la medicina herbaria, con enfermedades (interacciones fármaco- enfermedad) y, por supuesto, con otro fármaco, es decir, interacción fármaco-fármaco (drugdrug interactions o DDIs); este último tipo de interacción es motivo del presente artículo en el contexto de la práctica clínica geriátrica.

\section{Clasificación clínica de las interacciones fármaco-fármaco}

El espectro de las interacciones fármacofármaco es tan amplio que puede ir desde los que no tienen importancia clínica alguna o ausencia de reportes sobre alguna implicancia terapéutica, hasta aquellas interacciones que representan riesgo de reacción adversa severa para el paciente. La clasificación clínica de las interacciones medicamentosas más completa y práctica es la presentada por el Departamento de Farmacología del Hospital Huddinge de Estocolmo, Suecia $\left({ }^{4}\right)$ (Tabla 1). Esta clasificación permite identificar y seleccionar aquellas interacciones medicamentosas según su implicancia clínica y terapéutica. Las Tablas 2 y 3 señalan las principales y más frecuentes

Tabla 1.- Sistema de clasificación de interacciones medicamentosas de acuerdo a su relevancia clínica: Clasificación del Departamento de Farmacología del Huddinge Hospital, Stockholm *

\begin{tabular}{lll}
\hline $\begin{array}{l}\text { Categoría de interacción medicamentosa } \\
\text { (DDIs: drug-drug interactions) }\end{array}$ & \multicolumn{1}{c}{ Importancia clínica } & Ejemplo \\
\hline $\begin{array}{l}\text { Interacción medicamentosa } \\
\text { de tipo A(A-DDIs : A-drug } \\
\text { drug interactions) }\end{array}$ & Sin importancia clínica & \\
$\begin{array}{l}\text { Interacción medicamentosa de tipo B } \\
\text { (B-DDIs : B-drug drug interactions }\end{array}$ & $\begin{array}{l}\text { Efecto clínico de interacción } \\
\text { no ha sido establecido }\end{array}$ & Digitálicos / \\
$\begin{array}{l}\text { Interacción medicamentosa de tipo C } \\
\text { (C-DDIs : C-drug drug interactions) }\end{array}$ & $\begin{array}{l}\text { Posibles cambios en el efecto terapéutico } \\
\text { evitarse con ajuste de dosis de forma individual }\end{array}$ & Warfarina/AINEs \\
$\begin{array}{l}\text { Interacción medicamentosa de tipo D } \\
\text { (D-DDIs : D-drug drug interactions) }\end{array}$ & $\begin{array}{l}\text { Efectos adversos severos, ausencia de } \\
\text { efectos terapéuticos o ajuste de dosis } \\
\text { individuales son difíciles. Se recomienda }\end{array}$ & \\
& evitar la asociación de dichos fármacos & \\
\hline
\end{tabular}

\footnotetext{
* FASS (Pharmaceutical Specialities in Sweden). Stockholm: INFO Lakemedelsinformation AB (Drug information), 1997. URL: http://www.fass.se (Swedish).
} 
interacciones medicamentosas en adultos mayores que necesitan de regulación de dosis y las que deben ser evitadas; éstas son denominadas interacciones fármaco-fármaco de tipo C (C-drug-drug interaction o C-DDIs) y las interacciones fármaco-fármaco de tipo D (Ddrug-drug interaction o D-DDIs), respectivamente; estas últimas representan serios riegos para el paciente adulto mayor $\left({ }^{4}\right)$.

\section{Farmacoepidemiología}

En el análisis matemático, si un paciente está tomando 5 medicamentos, existe la probabilidad de $50 \%$ de una interacción importante clínicamente. Y, cuando son 7 los fármacos por paciente, la posibilidad se incrementa al $100 \%$; $20 \%$ de ellos podrían ser con manifestaciones de reacciones adversas severas $(5,6)$.

En el mundo real, las interacciones medicamentosas son causantes de $4,4 \%$ de todas las hospitalizaciones atribuidas a fármacos $\left({ }^{7}\right)$ y representan $4,6 \%$ de todas las reacciones adversas medicamentosas (RAM) en pacientes hospitalizados $\left({ }^{8}\right)$. En un estudio prospectivo con 1000 ancianos, se observó que 6\% de las hospitalizaciones fueron atribuidas a las interacciones fármaco-fármaco y más del 12\% tenían interacciones farmacológicas significativas al momento del internamiento $\left({ }^{9}\right)$. En otro estudio del año 2001 con pacientes mayores de 65 años en las salas de emergencias, la incidencia de potenciales interacciones fármaco-fármaco (utilizando un programa de computadora especialmente diseñado) fue de $31,1 \%$. En este mismo estudio se detectó $10,6 \%$ de reacciones adversas medicamentosas, ninguna de los cuales estuvo relacionada a las interacciones medicamentosas detectadas $\left({ }^{10}\right)$.

La prevalencia de interacciones farmacológicas en pacientes adultos mayores ambulatorios fue estudiado por Bjorkamn y el Pharmaceutical Care of the Elderly in Europe Research (PEER) Group ( ${ }^{11}$ ). En dicho estudio, donde el promedio de fármacos por paciente fue de 7 , se encontró que $46 \%$ de los 1601 ancianos de 6 países europeos tenían al menos una combinación de fármacos que podía generar una interacción medicamentosa de importancia clínica. Aproximadamente $90 \%$ de estas interacciones farmacológicas fueron clasificadas como interacciones medicamentosas de tipo $\mathrm{C}$, es decir, aquellas que requirieron ajuste de dosis para evitar efectos adversos. La Tabla 2 muestra las 10 interacciones medicamentosas de tipo $\mathrm{C}$ más frecuentes. Puede observarse que realmente son sólo 5 clases de fármacos que pueden presentar interacciones tipo C: diuréticos, inhibidores ECA, digitálicos, antiinflamatorios no esteroideos (y aspirina) y betabloqueadores. E1 $10 \%$ de potenciales interacciones medicamentosas fueron de tipo D, o sea, los que deben ser evitados por representar serio peligro de reacción adversa o ausencia de efectos terapéuticos. En la Tabla 3 están las 10 principales denominadas interacciones medicamentosas de tipo D que deben ser evitadas en adultos mayores. Notar que la interacción medicamentosa de tipo D más frecuente es la asociación entre bromuro de ipratropio y

Tabla 2.- Diez interacciones medicamentosas más frecuentes en adultos mayores que requieren necesariamente ajuste de dosis (interacción medicamentosa de tipo C) *

\begin{tabular}{lc}
\hline Fármacos & $\begin{array}{c}\text { Frecuencia(\%) } \\
(\mathrm{n}=1601)\end{array}$ \\
\hline Digoxina / Diuréticos & 13,6 \\
Diuréticos / AINEs & 9,6 \\
Furosemida / Inhibidores ECA & 9,4 \\
Inhibidores ECA / Dosis bajas de ASA & 7,0 \\
Inhibidores ECA / AINEs o dosis altas de ASA & 5,0 \\
Betabloqueadores / AINEs & 4,3 \\
Digitálicos / Verapamilo & 3,1 \\
Diuréticos / Sotalol & 1,7 \\
Codeína, combinaciones / antidepresivos & 1,6 \\
\hline
\end{tabular}

* Bjorkman IG, Fasbom J., Schmidt I, Bernsten CB y Pharmaceutical Care of the Elderly in Europe Research (PEER) Group. Drug-drug Interactions in the Elderly. Ann Pharmatother 2002; 36: 1675-81. 
Tabla 3.- Diez interacciones medicamentosas más frecuentes en adultos mayores que deben ser evitadas (interacción medicamentosa de tipo D) *

\begin{tabular}{cc}
\hline Fármacos & $\begin{array}{c}\text { Frecuencia } \\
\text { (total D-DDIs=129 } \\
\text { total pacientes }=1601) \\
\mathrm{n}\end{array}$ \\
\hline
\end{tabular}

Bromuro ipratropium / Agonistas selectivos

de los Beta $_{2}$ adrenoreceptores

Cloruro de potasio / Agentes ahorradores de potasio

Agentes antitrombóticos (warfarina,ticlopidina) /

AINEs o dosis altas de ASA

23

Agentes antitrombóticos (warfarina,ticlopidina) /

propafenona, amiodarona o cimetidina

Codeína, combinaciones / antipsicóticos

Metotrexate / ASA o AINEs

Verapamilo / beta bloqueadores

Verapamilo / beta bloqueadores (gotas oftálmicas)

Varias combinaciones (ASA / acetazolamida,

antiácidos o quinapril / tetraciclinas o norfloxacino, antiácidos / ácido ursodeoxicólico, cimetidina /

teofilina, dextropropoxifeno / alprazolam,

cimetidina / metformina, propafenona / metoprolol

* Bjorkman IG, Fasbom J., Schmidt I, Bernsten CB y Pharmaceutical Care of the Elderly in Europe Research (PEER) Group. Drug-drug Interactions in the Elderly. Ann Pharmatother 2002; 36: 1675-81

agonistas beta ${ }_{2}$, cuyo riesgo es muy alto en pacientes con predisposición para glaucoma de ángulo estrecho. De manera general, según el estudio de Bjorkman y col., 50\% de las interacciones farmacológicas detectadas resultaron en una reacción adversa; y, el otro $50 \%$, en un efecto terapéutico subóptimo $\left({ }^{11}\right)$.

La polifarmacia en el adulto mayor es otro factor de riesgo para las interacciones medicamentosas de tipo C y D. Según el estudio de Kurfees y col., en pacientes mayores de 60 que estaban tomando dos o más medicamentos (32\% de ellos tenía polifarmacia mayor, o sea, tomaba 5 ó más fármacos por paciente), se detectó un $42 \%$ de potenciales interacciones medicamentosas, de las cuales $27 \%$ fueron categorizadas como potencialmente peligrosas o serias $\left({ }^{12}\right)$.

\section{Superfamilia del citocromo $\mathbf{P 4 5 0}$}

Para poder predecir una interacción medicamentosa, es necesario conocer las enzimas responsables de la biotransformación del fármaco en el organismo. De las múltiples enzimas, la familia de citocromo P450 es la más importante en el metabolismo de los medicamentos. Entre ellas debe mencionarse a la CYP1A1, CYP2C, CYP2D6, CYP2E1 y CYP3A4. La última de ellas -CYP3A4- está implicada en cerca de $50 \%$ de los fármacos que se comercializa actualmente. Los fármacos que son conocidos por ser potentes inhibidores del citocromo $\mathrm{P} 450$ son amiodarona, fluoxetina, fluvoxamina, itraconazol, ketoconazol, omeprazole, quinidina y ritonavir. Sin embargo, existen también inductores enzimáticos que conducen a una disminución en la respuesta terapéutica de la droga, por ejemplo, los inductores del CYP3A4, como carbamazepina, dexametasona, fenobarbital, fenitoína, etosuximida, rifampicina y troglitazona $\left({ }^{13}\right)$.

\section{¿Cuándo una interacción fármaco-fármaco se hace clínicamente relevante?}

Descontando las interacciones medicamentosas beneficiosas, a pesar de existir en teoría un sinnúmero de interacciones, no todas son relevantes o se manifiestan clínicamente como un problema relacionado a medicamentos (PRM) que es necesario conocer. Los factores que actúan para que una interacción entre fármacos sea clínicamente relevante son los siguientes:

1) Índice o rango terapéutico del fármaco: amplio rango terapéutico permite grandes variaciones plasmáticas sin producción de reacciones adversas medicamentosas (RAMs). Los medicamentos con un estrecho rango terapéutico (relación de toxicidad y concentración mínima efectiva menor de 2 a 1 ó menos) son más susceptibles de producir RAMs originados por una interacción fármaco-fármaco. 
2) Afinidad de la enzima al fármaco: una alta afinidad previene el desplazamiento por otro fármaco.

3) Dosis utilizada: altas dosis de uno de los fármacos requieren dosis aun mayores del otro medicamento interactuante, para producir un efecto de desplazamiento.

4) Factores relacionados al paciente: edad, sexo, enfermedad, polifarmacia, etc.

Los fármacos en los cuales se ha reportado graves RAMs por interacciones medicamentosas están consignados en la Tabla 4.

\section{Las diez interacciones farmacológicas más frecuentes en geriatría (top ten dangerous drug interactions in long-term care)}

El tema de las interacciones medicamentosas es un tópico tan amplio, que incluso existen programas informáticos exclusivamente diseñados para indagar una posible interacción entre los medicamentos prescritos. La complejidad del tema, poco acceso a los programas mencionados, son quizás las causas por las que en la práctica clínica diaria es dejado de lado. Una de las estrategias para siempre tomar en cuenta es la de tener en mente sólo las principales y más frecuentes interacciones en el adulto mayor. Precisamente, éste fue el objetivo del Multidisciplinary Medication Management Project $\left({ }^{14}\right)$, que logró clasificar las "10 interacciones farmacológicas más frecuentes en centros de estancia prolongada de adultos mayores (top ten dangerous drug interactions in long-term care)". Esta lista es tan práctica, que bien puede ser usada como un tamizaje inicial para detectar una interacción farmacológica en otros niveles de atención geriátricos (Tabla 5).

El objetivo de la lista de las 10 interacciones farmacológicas más frecuentes en geriatría es la de convertirse en un instrumento útil para recordar que, cuando estemos frente a un paciente que esté recibiendo warfarina, inhibidores ECA, digoxina o teofilina, antes de prescribir un fármaco adicional debe tenerse presente la posibilidad de interacción medicamentosa. Dicho de otra manera, un

Tabla 4.- Fármacos metabolizados por el citocromo P450 con estrecho rango terapéutico e implicadas frecuentemente en reacciones adversas medicamentosas (RAM) severas *

\begin{tabular}{ll}
\hline Clase & Fármacos \\
\hline
\end{tabular}

Antiarrítmicos

Anticoagulantes

Anticonvulsivantes

Antidepresivos

Antihistamínicos

Antipsicóticos

Broncodilatadores

Hipoglicemiantes

Benzodiazepinas

Quimioterápicos

Hipolipemiantes Inmunosupresores

Inhibidores de la proteasa

Inhibidores de la transcriptasa reversa no nucleósidos Inhibidores de la transcriptasa reversa nucleosidos

Bloqueadores canales de calcio Miscelanea
Amiodarona, disopiramida, flecainida, mexiletine, propafenona, quinidina Warfarina

Carbamazepina, etosuximida, fenitoina, fenobarbital, ácido valproico Amitriptilina, amoxapina, clomipramina, desipramina, doxepin, fluoxetina, fluxovamina, imipramina, maprotilina, nortriptilina, paroxetina, protriptilina, sertralina, trimipramina Terfenadina, astemizole Clorpromazina, haloperidol, perfenazina, tioridazina Teofilina

Clorpropamida, gliclazida, glibenclamida, tolbutamida. Diazepam, midazolam, triazolam

Azatioprine, busulfan, doxoribicin, etoposide, ifosfamida, mercaptorurina. Fibratos, estatinas Ciclosporina, tacrolimus Indinavir, nelfinavir, ritonavir, saquinavir Efavirenz, delavirdine, devirapine

Didanosinezalcitabine, lamivudine, stavudine, zidovudine

Diltiazem, verapamilo Cisaprida, digoxina
* Souich P. In human therapy, is the drug-drug interaction or the adverse drug reaction the issue?.Can J Clin Pharmacol 2001; 3:153-161. 
Tabla 5.- Diez interacciones farmacológicas más frecuentes en centros de larga estancia de adultos mayores (top ten dangerous drug interactions in long-term care).

Las diez interacciones farmacológicas más frecuentes en geriatría*

\begin{aligned} & 1. Warfarina - AINEs \\ & 2. Warfarina - Sulfas \\ & 3. Warfarina - Macrólidos \\ & 4. Warfaina - Quinolonas \\ & 5. Warfarina - Fenitoína \\ & 6. Inhibidores ECA - Suplementos de potasio \\ & 7. Inhibidores ECA - Espironolactona \\ & 8. Digoxina - Amiodarona \\ & 9. Digoxina - Verapamilo \\ & 10. Teofilina - Quinolonas (ciprofloxacina, \\ & enoxacina, nofloxacina y ofloxacina) \\ & \hline\end{aligned}

* Multidisciplinary Medication Management Project Top Ten Dangerous Drug Interactions in Long-Term Care http:// www.amda.com/m3/topten.htm y http://www.scoup.net/ M3Project/topten/index.htm Accesado 1 febrero 2003.

paciente anticoagulado con warfarina, hipertensos en tratamiento con inhibidores ECA, pacientes con insuficiencia cardiaca usuarios de digoxina y pacientes con enfermedad pulmonar obstructiva crónica o asma usuarios de teofilina, deben ser tomados muy en cuenta para prevenir una interacción medicamentosa. La Tabla 6 aclara más detalles de la interacción y el manejo de los mismos.

Al igual que todos los problemas relacionados con medicamentos en pacientes geriátricos, las interacciones medicamentosas deben ser prevenidas, especialmente las que representan serios riesgos para el paciente, es decir, las del tipo D. En estos casos, el evitar la asociación de los fármacos es la mejor opción. En las interacciones medicamentosas de tipo C, la regulación de las dosis de los fármacos implicados prevendrá las reacciones adversas (Tabla 7).

En conclusión, las estrategias de prevención de reacciones adversas causados por interacciones medicamentosas, incluyen la identificación de pacientes en riesgo (pacientes adultos mayores usuarios de warfarina, digoxina, teofilina e inhibidores ECA) y, ante todo, paciente adulto mayor con polifarmacia. Categorizar y reconocer los tipos de interacción medicamentosa, teniendo muy en cuenta los de tipo C y D. Cuando se va agregar algún fármaco, hacerlo en lo posible a dosis graduales; y cuando se trate de fármacos nuevos en el mercado farmacéutico, debe estarse atento a los comunicados de los reportes de sitio en la web de MedWatch que alerten interacciones desconocidas hasta ese momento.

\section{REFERENCIAS BIBLIOGRÁFICAS}

1. Lasser KE, Allen PD y col. Timing of New Black Box warning and withdrawals for prescription medications. JAMA 2002;287:2215-20.

2. Federal Drug Agency USA. Addition to the list of drug products that have been withdrawn or removed form the market for reasons of safety or effectiveness. [Fecha de acceso 28 diciembre 2003]; Disponible en: http// www.fda.gov/cder/fdama/pcwdlist.txt.

3. Seymour RM, Routledge PA. Important drug-drug interactions the elderly. Drugs Aging 1998;12:485-94.

4. FASS (Pharmaceutical Specialities in Sweden). Stockholm: INFO Lakemedelsinformation AB (Drug information), 1997. [Fecha de acceso 25 marzo 2004]. Disponible en: http:// www.fass.se (Swedish).

5. Delafuente JC. Understanding and preventing drug interactions in elderly patients. Critical Rev Oncol/Hematol 2003;48:133-43

6. Karas Jr S. The potential for drug interactions. Ann Emerg Med 1981;10:627-30.

7. Stanton LA, Peterson GM, Rumble RH, Cooper GM, Polack AE. Drug related admissions to an australian hospital. J Clin Pharm Ther 1994;19:341-7.

8. Classen DC, Pestotnick SL, Evans RS, Lloyd JF, Burke JP. Adverse drug events in hospitalized patients. JAMA 1997;277:301-6.

9. Doucet J, Chassagne P, Trivalle C, Landrin I, Pauty MD, Kadri N, et al. Drug-drug interactions related to hospital admissions in older adults: a prospective study of 1000 patients. J Am Geriatr Soc 1996;44:944-8.

10. Hohl CM, Dankoff J, Colacone A, Afilalo M. Polypharmacy, adverse drug-related events, and potential adverse drug interactions in elderly patients presenting to an emergency department. Ann Emerg Med 2001;38:66671. 
Tabla 6.- Manejo de las 10 interacciones farmacológicas más frecuentes en geriatría *

\begin{tabular}{lll}
\hline Fármacos & Riesgo & Mecanismo de Acción \\
\hline $\begin{array}{l}\text { Warfarina }- \\
\text { AINEs }\end{array}$ & $\begin{array}{l}\text { Hemorragia } \\
\text { digestiva alta }\end{array}$ & $\begin{array}{l}\text { AINEs: aumenta irritación gás } \\
\text { y erosión de capa protectora de } \\
\text { estómago }\end{array}$ \\
$\begin{array}{l}\text { Warfarina - } \\
\text { Sulfas }\end{array}$ & $\begin{array}{l}\text { Riesgo de } \\
\text { hemorragia por } \\
\text { aumento efectos } \\
\text { de warfarina }\end{array}$ & $\begin{array}{l}\text { Administración de antibiótico } \\
\text { afecta flora intestinal que } \\
\text { produce vitamina K }\end{array}$ \\
\hline
\end{tabular}

Warfarina -
Quinolonas
(ciprofloxacina,
enoxacina,
nofloxacina y
ofloxacina)

\section{Warfarina -}

Macrólidos

\section{Warfarina- \\ Fenitoina}

ECA -

Suplementos

de potasio

Inhibidores

ECA -

Espironolactona

Digoxina -

Amiodarona
Riesgo de Administración de antibiótico hemorragia por afecta flora intestinal que produce aumento efectos de warfarina

\section{Riesgo de} hemorragia por aumento efectos de warfarina

\section{Incremento de} efectos de warfarina $y$ fenitoína

Hiperkalemia

Disminución de producción de aldosterona y disminución de excreción de potasio por vía renal

Hiperkalemia

Efecto aditivo de ambos fármacos que disminuyen excreción de potasio por vía renal

Intoxicación digitálica

Amiodarona disminuye depuración de digoxina. Efecto aditivo sobre nódulo sinusal cardiaco

$\begin{array}{lll}\begin{array}{l}\text { Teofilina- } \\ \text { Quinolonas }\end{array} & \begin{array}{l}\text { Intoxicación } \\ \text { por teofilina }\end{array} & \begin{array}{l}\text { Quinolonas inhiben } \\ \text { metabolismo hepático } \\ \text { de teofilina }\end{array}\end{array}$

$\begin{array}{ll}\text { Digoxina - } & \text { Intoxicación } \\ \text { Verapamilo } & \text { digitalica }\end{array}$

\section{Efecto sinergístico,} disminuyendo conducción y contractilidad muscular, llevado a bradicardia y bloqueo cardiaco

enoxacina, norfloxacina)

Prevención y alternativas Tratamiento

Evitar uso. Usar acetaminofen a dosis $<2 \mathrm{~g} /$ día no afecta INR. Discutible uso de inhibidores de COX-2 por reportes de elevación de INR.** Uso de tramadol

Evitar uso (especialmente sulfametoxasoltrimetropin). Si es muy necesario el uso de sulfametaxsol-trimetropin, reducir dosis warfarina al $50 \%$ durante terapia antibiótica. Monitorizar INR interdiario

Evitar uso (ciprofloxacina, enoxacina, nofloxacina y ofloxacina). Si es muy necesario el uso de auinolonas: (ciprofloxacina, enoxacina, nofloxacina y ofloxacina), monitorizar INR interdiario y ajustar dosis de warfarina

Evitar uso. Interacción puede ser de efecto prolongado. Usar otro grupo antibiótico si terapia antiinfecciosa es necesaria

Obtención de niveles basales de fenitoína sérica previo al inicio de warfarina. Monitorizar INR (Ideal nivel inferior de rango terapéutico)

Determinación de niveles séricos de potasio previo a inicio de inhibidor ECA

Al iniciar inhibidor ECA en un paciente, valorar niveles de potasio sérico. Evitar suplementos de potasio

Antes de iniciar amiodarona medir niveles séricos de digoxina, disminuir al 50\% dosis digoxina. Monitorizar digoxina sérica cada semana por varias semanas

Evaluar necesidad de Verapamilo (en pacientes con insuficiencia cardiaca, verapamilo no reduce morbilidad ni mortalidad)

\footnotetext{
Medir teofilina sérica previa a iniciación de la quinolona. Ciprofloxacina y enoxacina reducen la depuración de teofilina en $30-84 \%$. Considerar cambio de clase de antibiótico.
}

Monitorización de INR Manejo de hemorragia digestiva alta

Monitorizaciónde INR interdiario. Observación de signos de sangrado activo

Monitorización de INR interdiario. Observación de signos de sangrado activo

Si es muy necesario el uso de macrólidos, monitorizar INR y ajustar dosis de warfarina. Observación de signos de sangrado activo

Monitorización de niveles séricos defenitoína e INR. Observación de signos de sangrado activo

Monitorización de potasio sérico y unción renal. Manejo de Hiperkalemia

Monitorización de potasio sérico y función renal. Manejo de hiperkalemia

Mantener digoxina sérica entre 1 y 2 . Vigilar signos deintoxicación digitálica (en ancianos: dolor abdominal,anorexia,delirium)

Monitorizar frecuencia cardiaca y intervalo PR en el EKG. Vigilar signos de intoxicación digitálica(en ancianos: dolor abdominal, anorexia, delirio)

Monitorización de teofilina sérica, mantener en 5-15 $\mathrm{mcg} / \mathrm{mL}$. Aunque puede haber toxicidad por teofilina incluso en estos rangos. Toxicidad digitálica:convulsiones, náusea y vómitos

\footnotetext{
* Cuadro adaptado de: Multidisciplinary Medication Management Project Top Ten Dangerous Drug Interactions in Long-Term Care http://www.amda.com/m3/topten.htm y http://www.scoup.net/M3Project/topten/index.htm Accesado 1 febrero 2004

* Schaefer MG, Plowman BK, Morreale AP, Egan M. Interaction of rofecoxib and celecoxib with warfarin. Am J Health Syst Pharm. 2003 Jul 1;60(13):1319-23 (Medline)

* Mersfelder TL, Stewart LR. Warfarin and celecoxib interaction. Ann Pharmacother. 2000 Mar;34(3):325-7(Medline).
} 
Tabla 7.- Medidas de prevención de problemas relacionados con medicamentos causados por interacciones fármaco-fármaco en adultos mayores.

1. Identificar paciente en riesgo: En pacientes adultos mayores con medicación habitual de warfarina, inhibidores ECA, digoxina o teofilina, antes de agregar algún fármaco tener en cuenta la posibilidad de interacción fármaco-fármaco.

2. Identificar tipo de interacción fármaco-fármaco: En el caso de encontrar un interacción tipo C o tipo D, regular la dosis o cambiar uno de los fármacos respectivamente, para evitar reacciones adversas.

3. Dosis graduales: Iniciar en forma gradual fármacos que potencialmente pudieran causar una interacción fármaco-fármaco.

4. Precaución con fármacos nuevos en adultos mayores: Al prescribir un fármaco de reciente aparición en el mercado (menos de 7 años) siempre considerar la posibilidad de interacción fármaco-fármaco no reportados pero potencialmente severos. Los diseños de los estudios premarketing y la escasez de estudios postmarketing hacen difícil su detección en pacientes adultos mayores $\left({ }^{1}\right)$. La experiencia señala que la exposición a gran número de pacientes recién revelará las interacción fármaco-fármaco que muchas veces son graves y que finalmente hará que se retire el medicamento del mercado $\left.{ }^{(}\right)$. Siempre estar alerta a los informes en el sitio web de MedWatch y FDA (http//www.fda.gov).

5. Recordar si una interacción fármaco-fármaco no ha sido reportada no quiere decir que no exista. La grave interacción digoxina y quinidina fue descubierta recién en 1978, a pesar de haberse estado utilizando dichos fármacos por cerca de 30 años $\left({ }^{15-17}\right)$

11.Bjorkman IG, Fasbom J, Schmidt I, Bernsten CB, Pharmaceutical Care of the Elderly in Europe Research (PEER) Group. Drug-drug interactions in the elderly. Ann Pharmatother 2002;36:1675-81.

12. Kurfees JF. Dotson RL. Drug interactions in the elderly. J Fam Pract 1987;25:477-88.

13. Souich P. In human therapy, is the drug-drug interaction or the adverse drug reaction the issue?.Can J Clin Pharmacol 2001;3:153-61.

14. Brown KE. Multidisciplinary Medication Management Project: Top Ten Dangerous Drug Interactions in Long-
Term Care. 2003 [Fecha de Acceso: 1 febrero 2004]; Disponible en: http://www.amda.com/m3/topten.htm y http://www.scoup.net/M3Project/topten/index.htm

15. Leahey EB Jr, Reiffel JA, Drusin RE, Heissenbuttel RH, Lovejoy WP, Bigger JT. Interaction between quinidine and digoxin. JAMA 1978;240:533-4.

16. Ejvinsson G. Effect of quinidine on plasma concentrations of digoxin. Br Med J 1978;1:279-80.

17. Hooymans PM, Merkus FW. Effect of quinidine on plasma concentrations of digoxin. Br Med J 1978;2:1022. 